\section{Discussion}

The response rate of the study (35\%), while low, is original papers average for postal surveys (Parten, 1950; Kerlinger, 1973), but does mean conclusions can only be tentative. Our population is also likely to be skewed towards those who use alternative therapies because they were responding to an article on self-help therapy. A large minority of our group used hypnotherapy (28\%), which is not widely used in other European countries (Fisher \& Ward, 1994). None admitted to using homeopathy, probably because we did not specifically ask about homeopathy.

Within our sample, self-reported duration of problem was not associated with treatment-seeking behaviour, but self-rated severity of problem did. The more severe anxiety sufferers had been treated by a psychiatrist or psychologist and had used alternative therapies. Our findings agree with those of Astin (1998), who found poorer health predicted more alternative therapy use in 1035 randomly selected individuals who had agreed to participate in mail surveys. Our findings also agree in two respects with those of Marks \& Herst (1970). First, of the 1200 people with agoraphobia surveyed the $5 \%$ with no past medical treatment for their phobia were no more severe than those treated by a GP for their phobia. These untreated 5\% were, however, more shy about confiding in anyone (not only doctors) and took longer to seek help from anyone not only for their phobia, but also for their physical illnesses - the present study did not examine the latter issue. The second similarity was that the people with agoraphobia in Marks \& Herst's study who had been treated by a GP for their phobia were less severe than those who had had psychiatric out-patient treatment for it.
Our findings in a selected sample suggest that more severe anxiety sufferers use both mental health services and alternative therapies more. In our sample over half of respondents (55\%) who had been treated by a psychiatrist or psychologist had also used alternative therapies. In view of these findings, as clinicians, we need to educate ourselves as to the alternative therapies available, their efficacy and side-effects and we need to actively ask patients about their use.

\section{References}

ASTIN, J. (1998) Why patients use alternative medicine: results of national study. Journal of the American Medical Association, 279, 1548-1553.

CONSUMERS'ASSOCIATION (1992)

Regulation of Practitioners of NonConventional Medicine. London: Consumers'Association.

EISENBERG, D., DAVIS, R., ETTNER, S., et al (1998) Trends in alternative medicine use in the United States,

1990-1997: Results of a follow-up national survey. Journal of the American Medical Association, 208, 1569-1575.

FISHER, P. \& WARD, A. (1994)

Complementary medicine in Europe. British Medical Journal, 309, 107-110. GOLDBECK-WOOD, S., DOROZYNSKI, A., GUNNAR LIEL., et al (1996) Complementary medicine is blooming worldwide. British MedicalJournal, 313, 131-133.

KERLINGER, F. N. (1973) Foundations of Behavioural Research. NewYork: Holt, Rinehart \& Winston

MARKS, I. M. \& HERST, E. R. (1970)A survey of 1200 agoraphobics in Britain Social Psychiatry, 5, 16-24.

PARTEN, M. (1950) Surveys, Pools, and Samples: Practical Procedures. New York: Harper \& Row.

*Candida Graham Locum Consultant Psychiatrist, Abigail Franses Research Associate, South London and Maudsley NHS Trust, Maudsley Hospital, London SE5 8AZ, Mark Kenwright NurseTherapist, Isaac Marks Professor of Experimental Psychopathology, Institute of Psychiatry, De Crespigny Park, London SE5 8AF

\title{
Survey of staff perceptions of illicit drug use among patients in a medium secure unit
}

\author{
AIMS AND METHOD \\ A survey of staff perceptions of illicit \\ drug use among in-patients in a \\ medium secure unit. \\ RESULTS \\ Sixty per cent of staff were aware of \\ drug misuse on the unit. While $82 \%$ \\ commented on the potential adverse \\ effects of illicit substances on patient \\ psychopathology, $45 \%$ were also \\ concerned about the negative impact \\ on the staff-patient relationships. \\ Most (82\%) staff cite personal enjoy- \\ ment as the main reason for drug- \\ taking behaviour and perceive drug \\ use on the unit as a form of antisocial \\ behaviour. Less than one-third of \\ staff were clear about the unit's \\ policy for dealing with in-patient \\ drug use and few have had adequate \\ training in the management of \\ patients with dual diagnoses.
}

Strategies for dealing with drug misuse appear to focus on security rather than therapeutic issues.

\section{CLINICAL IMPLICATIONS}

Services need to provide adequate training for staff on the management of patients with comorbid substance misuse, introduce patient education programmes and develop and disseminate clear policies that emphasise both therapy and security.
Studies of psychiatric patients indicate a high rate of substance misuse among those diagnosed with schizo- phrenia (Breakey et al, 1974; Barbee et al, 1989; Mueser et al, 1990; Mathers et al, 1991). In this group comorbid 
substance misuse has been shown to be associated with a poorer outcome in terms of hospitalisation (Haywood et al, 1995), violence (Cuffel et al, 1994), incarceration (Abram \& Teplin, 1991) and homelessness (Drake et al, 1991). In addition, research suggests that cannabis misuse results in an exacerbation of psychotic symptomatology, hostility and non-compliance with prescribed medication (Freed, 1975; Safer, 1987; Cuffel et al, 1993).

It is well recognised that illicit drug use is prevalent in psychiatric hospitals (Deahl, 1991; Sandford, 1995), prisons (Wallace, 1993) and special hospitals (Fallon Inquiry, 1999). In secure psychiatric settings drug misuse is perceived as a significant risk factor for violent incidents, yet there has been little systematic study of the extent of the problem or the policies for dealing with illicit drug use among patients.

This study examined staff perceptions of the extent and impact of illicit drug use in a medium secure unit (MSU) for mentally disordered offenders.

\section{Method}

\section{Experiment design}

A questionnaire survey was conducted to collect data on staff perceptions of illicit drug use among current inpatients on the Edenfield Centre MSU during July 1998. The questionnaire was based on a similar survey conducted in a special hospital setting (McKeown \& Liebling, 1995). All ward-based staff, including nurses, medical staff, psychologists, social workers and occupational therapists, were invited to participate. Subjects were asked to comment on the extent of illicit drug use among in-patients, the predominant drugs of misuse and the impact of illicit drug use on patients' mental state, ward atmosphere, staff-patient relationships and peer group interactions. In addition, subjects were asked to rate the likelihood of specific diagnostic groups being involved in drug use. Similar comments were invited about the likelihood that certain groups of patients (categorised on source of referral) misuse drugs. Unit policies on drug use and training needs for staff dealing with patients with comorbid substance misuse were explored.

\section{Results}

\section{General characteristics of respondents}

Sixty-two staff responded to the questionnaire (response rate $64 \%)$. The majority of returns were from nursing staff $(n=48)$. The remainder were from junior medical staff $(n=7)$, occupational therapists $(n=5)$ and social workers $(n=2)$. Of those not responding, senior nursing staff were overrepresented.

\section{Extent and impact of drug use on the unit}

The majority of staff $(43,69 \%)$ reported that over half of the patients on the unit had a comorbid diagnosis of drug misuse. The remainder estimated that between 5 and $40 \%$ had a history of drug use.

Thirty-seven (60\%) staff were aware of in-patient illicit drug use. Sixty-one per cent were also aware of trafficking in prescribed drugs, particularly procyclidine. The most commonly reported illicit drugs coming into the unit were cannabis (92\%) and amphetamines (54\%). Seven subjects reported knowing that heroin, and three that cocaine, had been brought in.

Examination of staffs' perceptions of the impact of illicit drug use on the unit indicated that 51 (82\%) staff felt that illicit drug use precipitated a relapse in patients with psychosis or an escalation in aggression. A further four $(6 \%)$ staff felt that some patients appeared calmer or less disturbed on drugs such as cannabis. The majority of staff $(47,76 \%)$ reported that illicit drug use had a significant impact on peer group interactions, with 21 (34\%) reporting increased bonding and alliance among drug misusers. Twenty-three (37\%) staff, however, commented that drug use on the unit resulted in an escalation of bullying and intimidation. Twenty-eight (45\%) felt that illicit drug use among in-patients had a profoundly negative impact on staff-patient relationships, with resultant increases in hostility from patients, reduced engagement in therapy and increased stress among staff.

Seven staff commented that some of the hostility from patients was generated because staff have to adopt a 'policing' role in the prevention of drug misuse on the unit. Examination of the impact of illicit drug use on ward atmosphere indicated that the majority of staff $(51,82 \%)$ believed that drug use resulted in an unpleasant atmosphere, with descriptions such as "strained", "tense" or "disturbed". Five (8\%) staff, however, reported that drug use on the ward led to improvement in ward atmosphere because of the calming effects of drugs such as cannabis on some patients.

\section{Staff perceptions of patient groups likely to misuse drugs}

Staff were asked to rate the likelihood of patients from different referral sources being involved in in-patient drug use on a scale from one (very unlikely) to five (very likely). The mean ratings are shown in Table 1. Thirty-eight (61\%) respondents rated patients transferred from a prison setting as being more likely to use drugs on the unit. Patients transferred from district general and special hospitals were perceived to be somewhat less likely to use drugs during their in-patient stay. On a similar scale, patients with a primary diagnosis of personality disorder were considered marginally more likely to be involved in drug use than those with a diagnosis of mental illness. The mean scores were 3.64 and 3.48, respectively, but were not significantly different.

Ninety-eight per cent of respondents believed that friends and relatives of patients were responsible for bringing drugs onto the unit. The remainder believed that patients smuggled them in following community leave. 


\section{Perceived reasons for drug misuse}

Staff were asked to rate several predetermined reasons original for illicit drug use among patients on a scale of likelihood papers ( $1=$ very unlikely, to $5=$ very likely). Personal enjoyment (51, 82\%) was considered the most likely reason for drug use and the average ratings were high (Table 1). Escapism $(45,73 \%)$ was also considered a significant factor, while peer group pressures or self-medication were felt to be less common reasons for drug misuse. Additional reasons given by staff included boredom (17, 27\%) and cultural identity $(5,8 \%)$.

\section{Unit policy on illicit drugs}

Twenty-four (39\%) respondents reported that the unit had a policy of searching certain visitors' belongings. Eighteen (29\%) indicted that the unit had a policy for conducting random room searches and 16 (26\%) reported a policy for random urine drug screens. Fortyone $(66 \%)$ staff felt that the police should be called if visitors were found with drugs and $33(53 \%)$ suggested that those found carrying drugs should be banned from visiting.

For patients found in possession of illicit substances, 25 (40\%) believed sanctions (e.g. loss of leave privileges) should be instituted. Nineteen (31\%) suggested that the police should be notified, 15 (24\%) reported that specialist drug education should be offered and 12 (19\%) believed that counselling should be offered by the ward staff.

On the issue of facilities and therapies for patients involved in drug misuse, 22 (35\%) respondents said there were no available facilities, $23(37 \%)$ reported that keyworker basic training and support was the primary resource and 15 (24\%) said that a referral to a drug unit could be made but rarely occurred because the latter service was primarily set up to treat opiate dependence. Training-needs analysis indicated that 30 (70\%) members of staff had not received any training on drug misuse or drug-related issues in patients with mental disorder.

\section{Comment}

The overall response rate to the survey was higher than that in the Ashworth Hospital study (McKeown \& Leibling, 1995). However, we found that the poorest response rate was from nursing staff, despite the relevance of the subject matter to their daily management of patients. Non-responders among nursing staff tended to be those in more senior managerial positions and may reflect a belief that illicit drug use at ward level is of less relevance to them than it is to the front-line staff. Given the finding that substantial numbers of front-line staff were not aware of, or were unclear about, the unit's policies on illicit drug use, it is clear that these protocols need to be reviewed.

This survey indicates that illicit and prescribed drug misuse is perceived as relatively common and that cannabis, amphetamines and procyclidine are the major drugs of misuse. The reported substance misuse rates and
Table 1. Likelihood of and reasons for in-patient drug misuse

\begin{tabular}{lll} 
& Mean & s.d. \\
\hline Source of referral & & \\
Prison (sentenced) & 3.86 & 1.7 \\
Prison (remand) & 3.73 & 1.9 \\
Special hospital & 2.98 & 0.9 \\
District general hospital & 3.82 & 1.2 \\
Community & 3.22 & 1.4 \\
Reason for drug misuse & & \\
Personal enjoyment & 4.36 & 1.5 \\
Escapism & 4.12 & 1.7 \\
Peer-group pressure & 3.81 & 1.1 \\
Addiction & 3.67 & 0.9 \\
Self-medication & 2.60 & 1.8
\end{tabular}

1. Scale: $1=$ very unlikely, $5=$ very likely.

predominant drugs of misuse were similar to those reported in previous studies of patients with a primary diagnosis of schizophrenia (Alterman et al, 1980; Galanter et al, 1988). Although staff appear to be aware of the affect of illicit substances on patients' psychopathology, they appear just as concerned about the impact of patients' drug-taking behaviour on staff-patient relationships, ward atmosphere and the risk of aggression. Similar concerns were apparent in the Ashworth Hospital study, and it seems that front-line staff in secure facilities are particularly worried about the culture that surrounds drug misuse and drug trafficking.

Staff's reports that patients transferred from prisons were more likely to misuse drugs than those transferred from other hospitals appear to reflect their belief that prisons have a greater problem with illicit drug use than secure psychiatric facilities. In the majority of cases staff believed that patients misused illicit drugs for personal enjoyment or escapism. This belief may explain why so many staff emphasise security-related strategies for dealing with illicit drug-taking behaviours in patients. The suggestion that the police should be involved in some cases also appears to support the notion that many staff see drug misuse in in-patient settings as a form of criminal activity rather than a comorbid psychiatric disorder.

We were surprised by the number of staff reporting that there were no facilities or resources available for the management of drug misuse, and the finding that primary nurses with little training were the sole providers of education and therapy for patients with dual diagnoses. The Edenfield Centre is situated on the same hospital site as the regional drug rehabilitation service, but it would seem that referrals to the drug service are largely reserved for opiate users. Given that a substantial number of staff feel they require training on drug-related issues, it would seem worthwhile to consider the introduction of a drug awareness and education programme to assist staff in the treatment of dual-diagnosis patients. 


\section{References}

ABRAM, K. M. \& TEPLIN, L. A. (1991) Co-occurring disorders among mentally ill jail detainees: implications for public policy. American

Psychologist, 46, 1036-1045.

ALTERMAN, A. J., ERDLEN, D. L., LAPORTE, D. J., et al (1980) Problem drinking in hospitalised schizophrenic patients. Addictive Behaviour, $\mathbf{5}$ 273-276.

BARBEE, J. G., CLARK, P. D., CRAPANZANO, M. S., et al (1989) Alcohol and substance abuse among schizophrenic patients presenting to an emergency psychiatric service. Journal of Nervous and Mental Disease, 177 400-407.

BREAKEY,W. R., GODDELL, H., LORENZ, P. C., et al (1974) Hallucinogenic drugs as precipitent of schizophrenia. Psychological Medicine, 4, 225-260.

CUFFEL, B. J., HEITHOFF, K. A. \& LAWSON, W. (1993) Correlates of patterns of substance abuse among patients with schizophrenia. Hospital Community Psychiatry, 44, 247-251.

-, SHUMWAY, M., CHOULJIAN,T. L., et al (1994) A longitudinal study of substance use and community violence in schizophrenia. Journal of Nervous and Mental Disease, 182, 704-708

DEAHL, M. (1991) Cannabis and memory loss. British Journal of Addiction, 86, 249-252.

DRAKE, R. E., MCLAUGHLIN, P., PEPPER, $B$., et al (1991) Dual diagnosis of major mental illness and substance disorder : an overview. New Directions for Mental Health Services, 50, 3-12.

FALLON INQUIRY (1999) The Committee of Inquiry into the Personality Disorder Unit at Ashworth Hospital (CM4194-11). London: The Stationery Office.

FREED, E. X. (1975) Alcoholism and schizophrenia: the search for perspectives. Journal of Studies on Alcohol, 36, 853-881.
GALANTER, M., CASTANEDA, R. \& FERMAN, J. (1988) Substance abuse among general psychiatric patients: place of presentation diagnosis and treatment. American Journal of Drug and Alcohol Abuse $142,211-235$

HAYWOOD, TW. KRAVITZ, H. M. GROSSMAN, L. S., et al (1995) Predicting the 'revolving door' phenomenon among patients with schizophrenic, schizoaffective, and affective disorder. American Journal of Psychiatry, 152, 856-861.

MCKEOWN, M. \& LIEBLING, H. (1995)

Staff perception of illicit drug use within a special hospital. Journal of Psychiatric and Mental Health Nursing, 2, 343-350.

MATHERS, D. C., GHODSE, A. H CAAN, A.W., et al (1991) Cannabis use in a large sample of acute psychiatric admissions. British Journal of Addiction, 86, 779-784.

* Mairead Dolan Senior Lecturer in Forensic Psychiatry, Honorary Lecturer in Forensic Psychiatrist, Helen Kirwan Researcher, Edenfield Centre, Mental Health Services of Salford, Bury New Road, Prestwich, Manchester M25 3BL
MUESER, K. T., YARNOLD, P. R., LEVINSON, D. F., et al (1990) Prevalence of substance abuse in schizophrenia: demographic and clinical correlates. Schizophrenia Bulletin, 16, 31-55.

SAFER, D. J. (1987) Substance abuse by young adult chronic patients. Hospital and Community Psychiatry, 38 511-514.

SANDFORD,T. (1995) Drug use is increasing. (A recent Royal College of Nursing Survey suggests that illicit drug use is increasing among psychiatric patients.) Nursing Standard, 9, 16-17.

WALLACE, J. (1993) Treatment behind bars - a new initiative. Addiction Counselling World, 4, 4-7.

\title{
RITA M. CONDREN, JOHN O'CONNOR AND ROY BROWNE
}

\section{Prevalence and patterns of substance misuse in schizophrenia}

\author{
A catchment area case-control study
}

\section{AIMS AND METHOD}

To identify the prevalence and patterns of substance misuse in patients with schizophrenia in a catchment population in Dublin, and to compare this with that of a control group from general practice. Ninety-nine patients and 75 controls were interviewed using a semi-structured technique. Urine samples were obtained from all patients and controls were analysed for illicit substances.

\author{
RESULTS \\ Prevalence of illicit substance and \\ alcohol misuse was similar among \\ patients (illicit substances, 45\%; \\ alcohol, 33\%) and controls (illicit \\ substances, $43 \%$; alcohol, $25 \%$ ). \\ Patterns of substance misuse were \\ similar, apart from ecstasy, which was \\ used more commonly by controls. \\ Discrepancies were found between \\ histories of drug use and urinalysis.
}

\begin{abstract}
CLINICAL IMPLICATIONS
Illicit substance misuse in patients with schizophrenia may mirror usage in the general population, rates of misuse reflecting cultural factors rather than illness and patterns of misuse reflecting geographical variations in the availability of drugs. A combination of screening methods is more effective than the use of a single source of information.
\end{abstract}

Comorbid psychotic illness and substance misuse has been the subject of increasing research and concern, mainly in the USA and more recently in Europe. Substance misuse has been reported to be the most prevalent comorbid condition associated with schizophrenia (Boyd et al, 1984; Regier et al, 1990). The Epidemiological Catchment Area (ECA) study revealed a life-time prevalence of $47 \%$ for substance misuse in patients with schizophrenia, of which $33.7 \%$ met criteria for an alcohol disorder and $27.5 \%$ for another drug misuse disorder (Regier et al, 1990). Patients with schizophrenia were 4.6 times more likely to have drug or alcohol problems than the general population. A number of investigators in Europe have found differing prevalence rates of comorbid psychotic illness and substance misuse, all less than that found in the ECA study (Bernadt \& Murray, 1986; Soyka et al, 1993; Duke et al, 1994; Hambrecht \& Hafner, 1996; Menezes et al, 1996). The widely differing reported 